\title{
Low-Level Laser Therapy Activates NF-kB via Generation of Reactive Oxygen Species in Mouse Embryonic Fibroblasts
}

\author{
Aaron C-H. Chen ${ }^{19}$, Praveen R. Arany ${ }^{1,2,3,49}$, Ying-Ying Huang ${ }^{1,5,6}$, Elizabeth M. Tomkinson ${ }^{1,7}$, Sulbha K. \\ Sharma ${ }^{1}$, Gitika B. Kharkwal ${ }^{1,5}$, Taimur Saleem ${ }^{1,8}$, David Mooney ${ }^{4}$, Fiona E. Yull ${ }^{9}$, Timothy S. Blackwell ${ }^{9}$, \\ Michael R. Hamblin ${ }^{1,4,10_{*}}$
}

1 Wellman Center for Photomedicine, Massachusetts General Hospital, Boston, Massachusetts, United States of America, 2 Programs in Leder Human Biology and Translational Medicine, and Biological Sciences in Dental Medicine, Harvard University, Cambridge, Massachusetts, United States of America, $\mathbf{3}$ Program in Oral and Maxillofacial Pathology, Harvard School of Dental Medicine and Brigham and Women's Hospital, Boston, Massachusetts, United States of America, 4 Harvard School of Engineering and Applied Sciences, Cambridge, Massachusetts, United States of America, 5 Department of Dermatology, Harvard Medical School, Boston, Massachusetts, United States of America, 6 Aesthetic and Plastic Center, Guangxi Medical University, Nanning, People's Republic of China, 7 Smith College, Northampton, Massachusetts, United States of America, 8 Aga Khan Medical College, Karachi, Pakistan, 9 Department of Medicine and Cancer Biology, Vanderbilt University School of Medicine, Nashville, Tennessee, United States of America, $\mathbf{1 0}$ Harvard-MIT Division of Health Sciences and Technology, Cambridge, Massachusetts, United States of America

\section{Abstract}

Background: Despite over forty years of investigation on low-level light therapy (LLLT), the fundamental mechanisms underlying photobiomodulation at a cellular level remain unclear.

Methodology/Principal Findings: In this study, we isolated murine embryonic fibroblasts (MEF) from transgenic NF-kB luciferase reporter mice and studied their response to $810 \mathrm{~nm}$ laser radiation. Significant activation of NF-kB was observed at fluences higher than $0.003 \mathrm{~J} / \mathrm{cm}^{2}$ and was confirmed by Western blot analysis. NF-kB was activated earlier ( 1 hour) by LLLT compared to conventional lipopolysaccharide treatment. We also observed that LLLT induced intracellular reactive oxygen species (ROS) production similar to mitochondrial inhibitors, such as antimycin A, rotenone and paraquat. Furthermore, we observed similar NF-kB activation with these mitochondrial inhibitors. These results, together with inhibition of laser induced NF-kB activation by antioxidants, suggests that ROS play an important role in the laser induced NF-kB signaling pathways. However, LLLT, unlike mitochondrial inhibitors, induced increased cellular ATP levels, which indicates that LLLT also upregulates mitochondrial respiration.

Conclusion: We conclude that LLLT not only enhances mitochondrial respiration, but also activates the redox-sensitive NFkB signaling via generation of ROS. Expression of anti-apoptosis and pro-survival genes responsive to NFkB could explain many clinical effects of LLLT.

Citation: Chen AC-H, Arany PR, Huang Y-Y, Tomkinson EM, Sharma SK, et al. (2011) Low-Level Laser Therapy Activates NF-kB via Generation of Reactive Oxygen Species in Mouse Embryonic Fibroblasts. PLoS ONE 6(7): e22453. doi:10.1371/journal.pone.0022453

Editor: Wafik S. El-Deiry, Penn State Hershey Cancer Institute, United States of America

Received February 10, 2011; Accepted June 28, 2011; Published July 21, 2011

Copyright: (c) 2011 Chen et al. This is an open-access article distributed under the terms of the Creative Commons Attribution License, which permits unrestricted use, distribution, and reproduction in any medium, provided the original author and source are credited.

Funding: This work was supported by National Institutes of Health (NIH) grant R01Al050875, Center for Integration of Medicine and Innovative Technology (DAMD17-02-2-0006), CDMRP Program in TBI (W81XWH-09-1-0514) and Air Force Office of Scientific Research (FA9950-04-1-0079). The funders had no role in study design, data collection and analysis, decision to publish, or preparation of the manuscript.

Competing Interests: The authors have declared that no competing interests exist.

* E-mail: hamblin@helix.mgh.harvard.edu

9 These authors contributed equally to this work.

\section{Introduction}

Low level light (or laser) therapy (LLLT) has been used for more than forty years to promote healing, reduce pain and inflammation, and prevent tissue death [1,2]. Despite many basic and clinical reports, the therapy remains controversial largely due to uncertainties about the fundamental molecular and cellular mechanisms responsible for transducing signals from the photons incident on the cells to the biological effects that take place in the irradiated tissues.

It has been reasonably well established that mitochondria are a principal intracellular target of red and near-infra-red light [3]. Cytochrome $\mathrm{C}$ oxidase (unit IV of the mitochondrial respiratory chain) is a chromophore that absorbs light as far into the infra-red as $1000 \mathrm{~nm}$ [4]. There have been reports of increased cytochrome c oxidase activity after LLLT [5] and many reports of increased ATP synthesis after light delivery to isolated mitochondria [6]. Additional evidence of the role of cytochrome c oxidase as a chromophore in LLLT has been provided by action spectra studies from Karu's laboratory in Russia [7] and from Eells and Wong-Riley in Wisconsin [8]. Many genes have their transcription upregulated (or down regulated) after illumination of cells with various wavelengths and fluences of light. For instance, illumination of human fibroblasts with $628 \mathrm{~nm}$ light emitting diode led to altered expression of 111 genes (68 up, 43 down) that can be sub-categorized into 10 functional groups [9]. 
Nuclear factor kappa B (NF-kB) is a transcription factor regulating expression of multiple genes [10], and has been shown to govern various cellular functions, including inflammatory and stress-induced responses and survival [11]. NF-kB activation is regulated by negative feedback mediated by $\mathrm{IkB}$, an inhibitor protein that binds to NF-kB, but can undergo ubiquitination and proteasomal degradation [12], thus freeing NF-kB to translocate to the nucleus and initiate transcription [13]. NF-kB is a redoxsensitive transcription factor [14], that has been proposed to be the sensor for oxidative stress [15]. Reactive oxygen species (ROS) can both activate NF-kB directly [16], and ROS are also involved in NF-kB activation by other stimuli such as tumor necrosis factor alpha (TNF $\alpha)$, phorbol ester, and interleukin (IL)-1 [17]. Several laboratories have observed the formation of ROS in cells in vitro after LLLT [18,19,20,21], and it has been proposed that ROS are involved in the signaling pathways initiated after photons are absorbed by the mitochondria within cells [22].

In the present report, we describe the effect of light from an $810 \mathrm{~nm}$ laser on mouse embryonic fibroblasts (MEF) isolated from a transgenic NF-kB luciferase reporter (HLL) mouse [23]. These mice have been genetically engineered so that luciferase expression is driven by the NF-kB-dependent portion of the human immunodeficiency virus-1 long terminal repeat. They have been used to carry out molecular imaging using a bioluminescence camera of inflammation after various stimuli such as tumor necrosis TNF $\alpha$, lipopolysaccharide (LPS) and IL-1 [24]. We reasoned that these cells would be ideal to test the hypothesis that LLLT activates NF-kB to mediate various downstream biological processes.

\section{Results}

\section{Activation of NF-kB by laser in vitro}

We used an agonist of toll-like receptor 4 signaling, the bacterial product lipopolysaccharide to confirm activation of NF-kB activation in the isolated primary MEFs. The results (Fig. 1A) show an increased NFkB expression at 6 hours following LPS addition that increased further till 10 hours before returning to baseline at 24 hours. We then irradiated cells with $0.3 \mathrm{~J} / \mathrm{cm}^{2}$ of $810 \mathrm{~nm}$ laser and measured luciferase response at the same time points (Fig. 1B). We observed a distinct NF-kB luciferase increase as early as 1 hour post-laser and the responses at 6, 10 and 24 hours were similar to those seen with LPS. The light fluence response of NF-kB measured at 6 hours is shown in Fig. 1C (solid line). We observed the strongest signal at 6 hours with $0.3 \mathrm{~J} / \mathrm{cm}^{2}$ but the luminescence decreased somewhat (but was still significantly above baseline) when the energy density was higher. To confirm that the luminescence increase measured was due to the increased expression of luciferase enzyme activated by NF-kB binding to the HIV long terminal repeat alone and not a direct effect of laser irradiation on the transgene, we pre-incubated Luc MEF with the protein synthesis inhibitor cycloheximide $(10 \mu \mathrm{M}$ for 2 hours) and then laser irradiated with same power densities and measured the luminescence at 6 hours. We observed that after cycloheximide, luminescence at all fluences was depleted to a steady level, about $30 \%$ of baseline level (Fig. 1C broken line).

$\mathrm{NF}-\mathrm{kB}$ activation proceeds via phosphorylation of its inhibitor, IKK $\alpha / \beta$ which leads to its ubiqitination and degradation, thus allowing phospharylation of the $\mathrm{p} 65$ subunit which then translocates to the nucleus to induce specific gene expression. To confirm that these biochemical pathways are activated by LLLT, we performed western blot analysis of MEF cell extracts at 5, 15 and 30 minutes after treatment with $0.3 \mathrm{~J} / \mathrm{cm}^{2}$ of $810-\mathrm{nm}$ laser, and with different fluences $\left(0.3,3\right.$, and $\left.30 \mathrm{~J} / \mathrm{cm}^{2}\right)$ at 15 minutes (Fig. 1D). We observed the distinct phosphorylation bands of both IKK $\alpha / \beta$ and NF-kB p65 as early as 5 minutes post light and were maintained for 30 minutes further validating the luciferase reporter results.

\section{Laser increases ROS production}

Many groups have reported the increase of cellular reactive oxygen species (ROS) after LLLT in vitro [19,25,26,27,28,29]. Since one of the important initiators of NF-kB activation in several cell types is known to be via ROS generation [15], we checked to if ROS were increased after laser irradiation at varying fluencies in our MEF NF- $\kappa$ B Luc cells using fluorescent reporters namely, CM- $\mathrm{H}_{2}$ DCFDA, MitoTracker and DAPI (Fig. 2A). While $0.03 \mathrm{~J} /$ $\mathrm{cm}^{2}$ gave little fluorescence above background (data not shown), $0.3,3$ and $30 \mathrm{~J} / \mathrm{cm} 2$ gave a robust increase in green fluorescence. Since the primary source of ROS within cells is superoxide generated in the mitochondria [30], we used the MitoTracker Red to demonstrate co-localization of the laser generated ROS with CM- $\mathrm{H}_{2}$ DCFDA as evidenced by the overlay (Fig. 2A). Quantitative validation of this result was performed with the Amplex Ultrared assay that demonstrated increased ROS generation at the three fluences used (Fig. 2B).

\section{Laser increases ATP synthesis}

To test if the NF-Kb Luc MEF respond to $810 \mathrm{~nm}$ laser irradiation with increased ATP, we first irradiated the cells with fluences varying over 5 orders of magnitude $\left(0.003\right.$ to $\left.30 \mathrm{~J} / \mathrm{cm}^{2}\right)$ and measured ATP levels over time. The results measured at 5 minutes post-light showed no increase with $0.003 \mathrm{~J} / \mathrm{cm}^{2}$, a small increase at $0.03 \mathrm{~J} / \mathrm{cm}^{2}$ and a large increase that formed a plateau with fluences of $0.3,3$ and $30 \mathrm{~J} / \mathrm{cm}^{2}$ (Figure 3A solid line). The time course of ATP increase reached a peak immediately after a 5-minute irradiation and gradually declined to baseline levels in 6 hours (Figure 3B). To confirm specificity, we pre-incubated the cells for 2 hours with sodium azide and deoxyglucose, widely used as a mitochondrial inhibitor and an inducer of chemical hypoxia [31], in the media and then performed laser irradiation and observed ATP generation. The metabolic inhibitors not only lowered the baseline levels of ATP but also made the cells unresponsive to the laser induced ATP generation (Fig. 3A dashed line).

\section{Mitochondrial inhibitors increase ROS and NF-kB activation but not ATP}

We tested three mitochondrial inhibitors that have all been reported to induce intracellular ROS by blocking the normal flow of electrons in the mitochondrial respiratory chain[32,33,34]. We reasoned that if the mechanism of LLLT activation of NF-kB was via generation of ROS, then these mitochondrial inhibitors should be able to activate NF-kB as well. We observed that all the inhibitors after 2 hour incubation showed NF-kB activation at the 6-hour time point (Figure 4A). Paraquat and rotenone showed similar NF-kB activation perhaps due to the fact that they are both complex I inhibitors, and antimycin A, a complex III inhibitor, induced the strongest activation. We also measured ATP changes in the cells with these inhibitors and found that, in contrast to $810 \mathrm{~nm}$ laser, they depleted ATP as might be expected from their ability to inhibit mitochondrial respiration by cutting electron transport at various sites (Figure 4B). Figure 4C-F confirms that these inhibitors increased ROS levels in MEF cells as determined by increased fluorescence intensity of CM- $\mathrm{H}_{2}$ DCFDA at $30 \mathrm{~min}$ following treatment.

\section{Antioxidants abrogate NF-kB activation but not ATP after laser}

To further confirm that the activation of NF-kB is due to lasergenerated mitochondrial ROS we pre-incubated the antioxidants, 

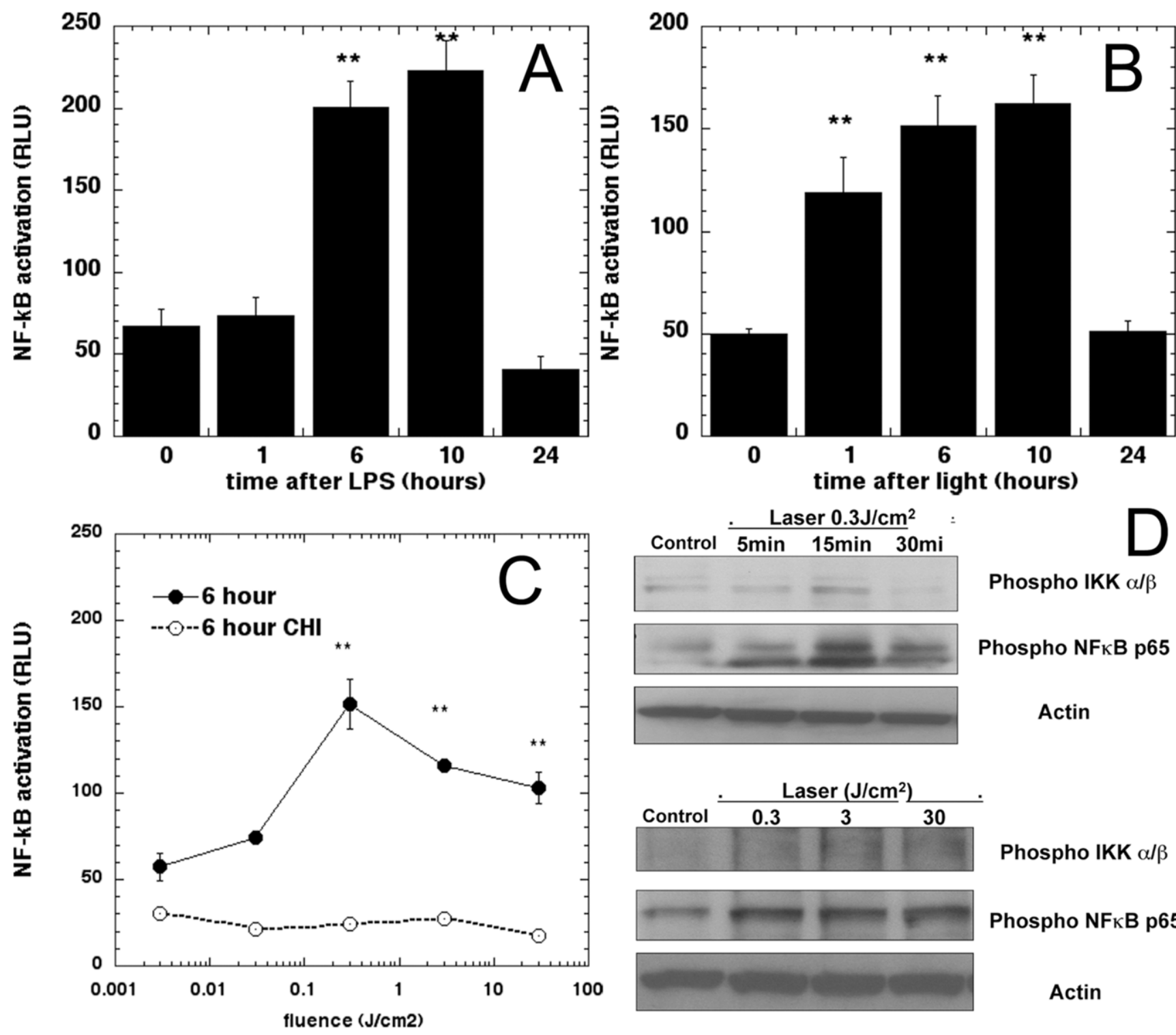

Phospho IKK $\alpha / \beta$

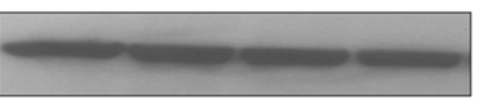

Phospho NFкB p65
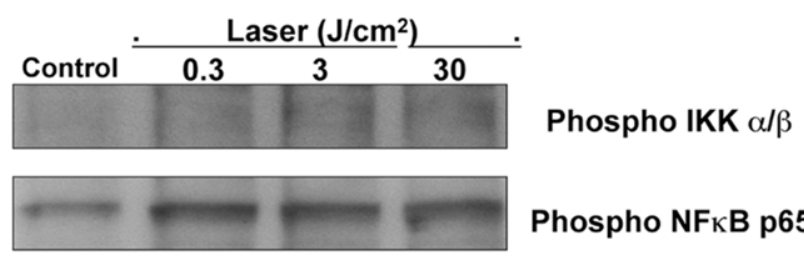

Phospho NFKB p65

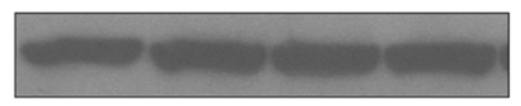

Actin

Figure 1. Laser activates NF-kB in MEF. (A) Time course of NF-kB activation measured by luminescence after addition of $0.5 \mathrm{ug} / \mathrm{mL}$ of LPS (B) Time course of NF-kB activation after $0.3 \mathrm{~J} / \mathrm{cm} 2$ 810-nm laser irradiation. (C) NF-kB measured 6 hours post irradiation with a wide range of $810-$ laser fluences and effect of protein synthesis inhibitor cycloheximide. ${ }^{*} p<0.05 ;{ }^{*} p<0.01$ compared to baseline. (D) Immunoblotting for Phospho NFKB and IKB $\alpha / \beta$ following laser irradiation of MEFs at $0.3 \mathrm{~J} / \mathrm{cm}^{2}$ over time (upper panel) and at various fluences $0.3,3 \mathrm{and} 30 \mathrm{~J} / \mathrm{cm}^{2}$ at $15 \mathrm{~min}$ (lower panel) was performed. Normalization for protein loading with Actin is shown.

doi:10.1371/journal.pone.0022453.g001

N-acetyl-L-cysteine (NAC) and ascorbic acid and performed LLLT and luciferase assays. The results shown in Fig. 5A showed that both ascorbate and to a lesser extent NAG significantly reduced the laser induced increase in NF-kB activation but had no significant effects on the control. We then asked whether the antioxidants had any effect on the laser-induced increase in ATP. As seen in Fig. 5B neither pre-incubation with NAC (nor ascorbate - data not shown) did not have any effect on the increased ATP generation following laser irradiation at $0.3 \mathrm{~J} / \mathrm{cm}^{2}$.

\section{Discussion}

This report has demonstrated for the first time that low levels of near-infrared laser light can activate NF-kB in primary MEF cells. We confirmed reports from other laboratories [19,25,26,27,28,29] that visible (and NIR light) can induce expression of ROS in illuminated cells. Our results indicate that activation of NF-kB after $810 \mathrm{~nm}$ laser light is mediated via ROS generation. The fact that the addition of antioxidants abrogates the activation of NF-kB provides additional evidence that ROS are involved in the activation of NF-kB. There are both similarities and differences between the cellular effects of NIR laser and inhibitors that produce mitochondrial ROS by inhibiting complexes of the respiratory chain. Both laser and mitochondrial inhibitors produce ROS and activate NF-kB, but laser increases ATP while the inhibitors decrease ATP. The fact that antioxidants do not abrogate the ATP increase suggests that the action of light increases electron transport, which in the absence of antioxidants can cause increased electron leakage producing superoxide.

The very wide range of fluences ( 3 orders of magnitude between 0.03 and $30 \mathrm{~J} / \mathrm{cm}^{2}$ ) that gave positive NF-kB activation suggests 

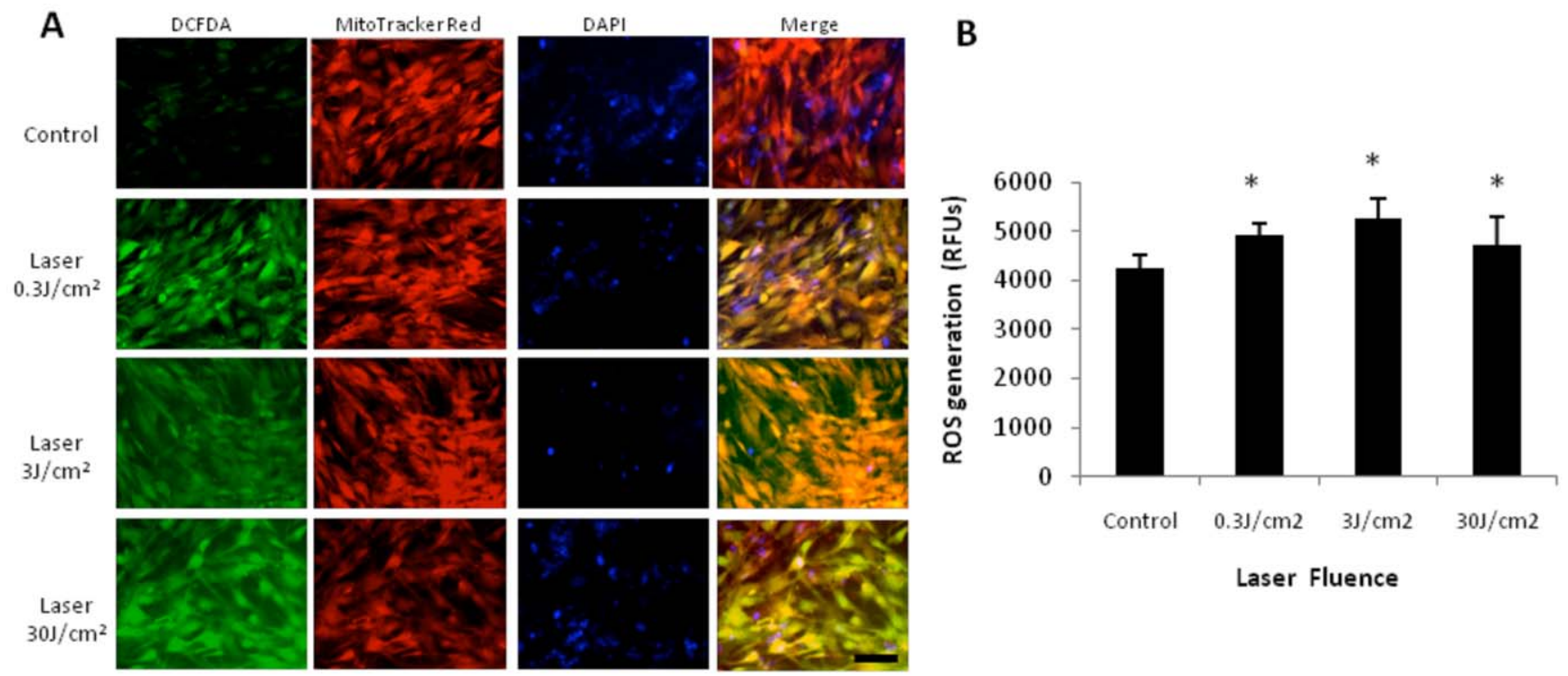

Control

\section{Laser Fluence}

Figure 2. Laser increases ROS in MEF. (A) MEF were treated with $810 \mathrm{~nm}$ laser at varying fluencies $0,0.3,3$ and $30 \mathrm{~J} / \mathrm{cm}^{2}$ and ROS generation was measured with $\mathrm{CM}-\mathrm{H}_{2}$ DCFDA (green). Mitochondrial localization is demonstrated with MitoTracker Red (red) and overlay (yellow) while cell nuclei were strained with DAPI (blue) (B) Amplex Ultrared assay demonstrates quantitative increase in ROS following laser irradiation at varying fluences. ${ }^{*} p<0.05$.

doi:10.1371/journal.pone.0022453.g002

that the phenomenon is akin to a switch being turned on after a minimum light dose in the region of $0.03 \mathrm{~J} / \mathrm{cm}^{2}$ has been delivered as has been observed with other LLLT induced biological processes such as latent TGF- $\beta 1$ activation [35]. The time course of the laser-induced NF-kB activation we observed appeared to be significantly different from that observed with conventional LPS activation [36]. Following laser irradiation, we observed significant activation at 1 hour while LPS did not show any significant increase until 6 hours. It has been recently discovered that there are (at least) two NF-kB activation pathways [37]. The canonical NF-kB signaling pathway activated in response to infections (toll like receptor signaling) and cytokines is based on degradation of $\mathrm{IkB}$ inhibitors. This pathway depends on the IkB kinase (IKK), which contains two catalytic subunits, $\operatorname{IKK} \alpha$ and IKK $\beta$. IKK $\beta$ is essential for inducible IkB phosphorylation and degradation, whereas IKK $\alpha$ is not. IKK $\alpha$ is involved in processing of the NF-kB2 (p100) precursor. IKK $\alpha$ preferentially phosphorylates NF-kB2, and this activity requires its phosphorylation by upstream kinases, one of which may be NF-kB-inducing kinase (NIK). IKK $\alpha$ is therefore a pivotal component of a second $\mathrm{NF}-\mathrm{kB}$ activation pathway based on regulated NF-kB2 processing rather than $\mathrm{IkB}$ degradation.
A

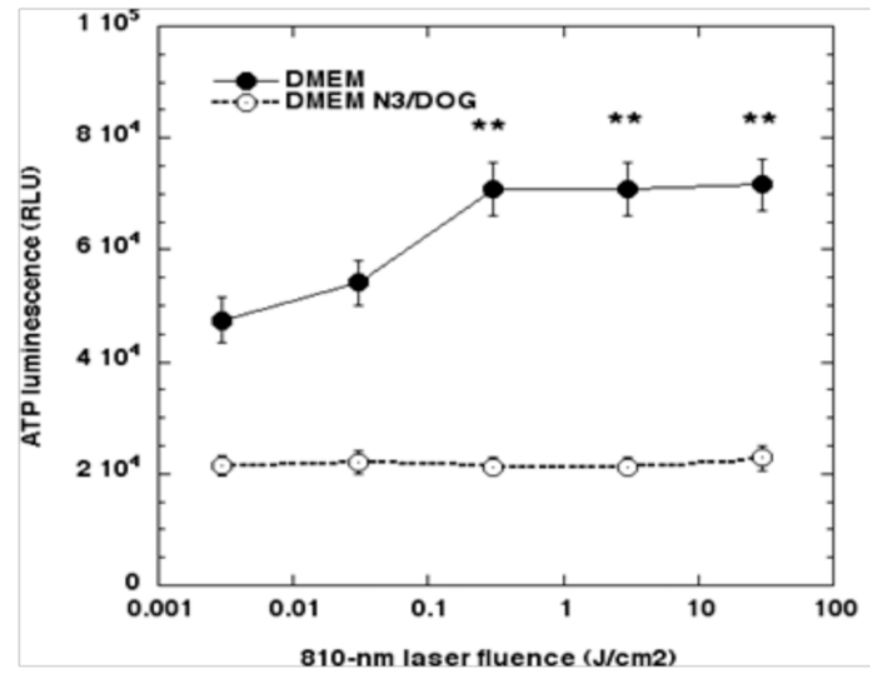

B

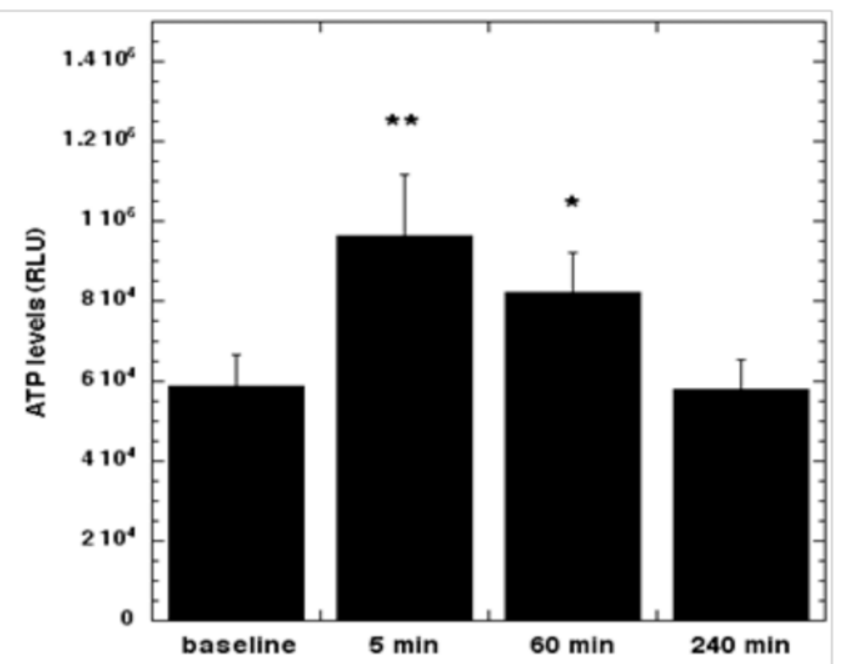

Figure 3. Laser increases ATP synthesis in MEF. (A) ATP increase measured at 5 min post LLLT with wide range laser fluences and effect of mitochondrial inhibitors (sodium azide/deoxyglucose). (B) Time course of ATP increase after $0.3 \mathrm{~J} / \mathrm{cm} 2810 \mathrm{~nm}$ laser. ${ }^{*} p<0.05 ;{ }^{* *} p<0.01$ compared to baseline.

doi:10.1371/journal.pone.0022453.g003 

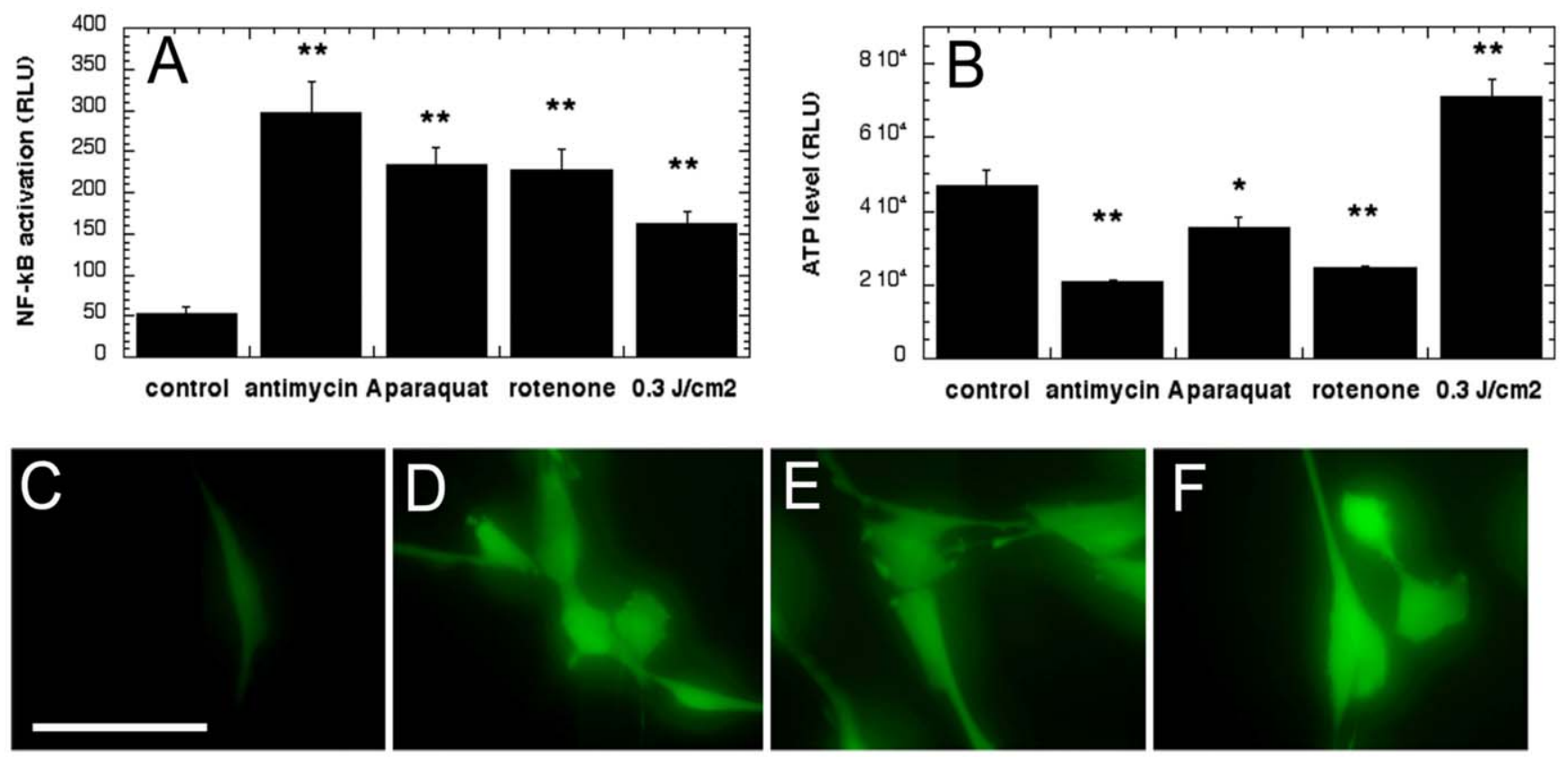

Figure 4. Mitochondrial inhibitors increase ROS and NF-kB activation in MEF but reduce ATP. (A) NF-kB activation measured at 6 hours after 2 hour incubation with $100 \mu \mathrm{M}$ inhibitors, (B) ATP levels measured after 2 hours incubation with $100-\mu \mathrm{M}$ inhibitor. ${ }^{*} \mathrm{p}<0.05 ;{ }^{*} \mathrm{p}<0.01$ compared to control. Inhibitors $(100 \mu \mathrm{M})$ were added for 2 hours and $\mathrm{CM}-\mathrm{H}_{2}$ DCFDA was used to measure ROS generation at 30 min (C) control, (D) antimycin $A,(E)$ rotenone, $(F)$ paraquat. doi:10.1371/journal.pone.0022453.g004

Recent work has identified protein kinase $\mathrm{D}$ (PKD, formerly known as PKCv) as a mitochondrial sensor of oxidative stress [38]. It was reported that low dose hydrogen peroxide (250-nM) led to tyrosine phosphorylation at Tyr463 on PKD and the enzyme's consequent activation [39]. This activation of PKD then led to activation of IKK $\beta$, followed by IkB degradation and NF-kB activation [40]. The pathway did not depend on IKK $\alpha$ nor on NF-kB inducing kinase (NIK) [39].
Considering the fact that NF-kB is known to be a redox-sensitive nuclear transcription factor [15] it is reasonable to conclude that laser induced NF-kB is due to the ability of the laser to produce mitochondrial ROS. The primary ROS generated in the mitochondrial electron transport chain is superoxide, which is destroyed by superoxide dismutase (SOD). SOD converts superoxide to a freely diffusible molecule, hydrogen peroxide that
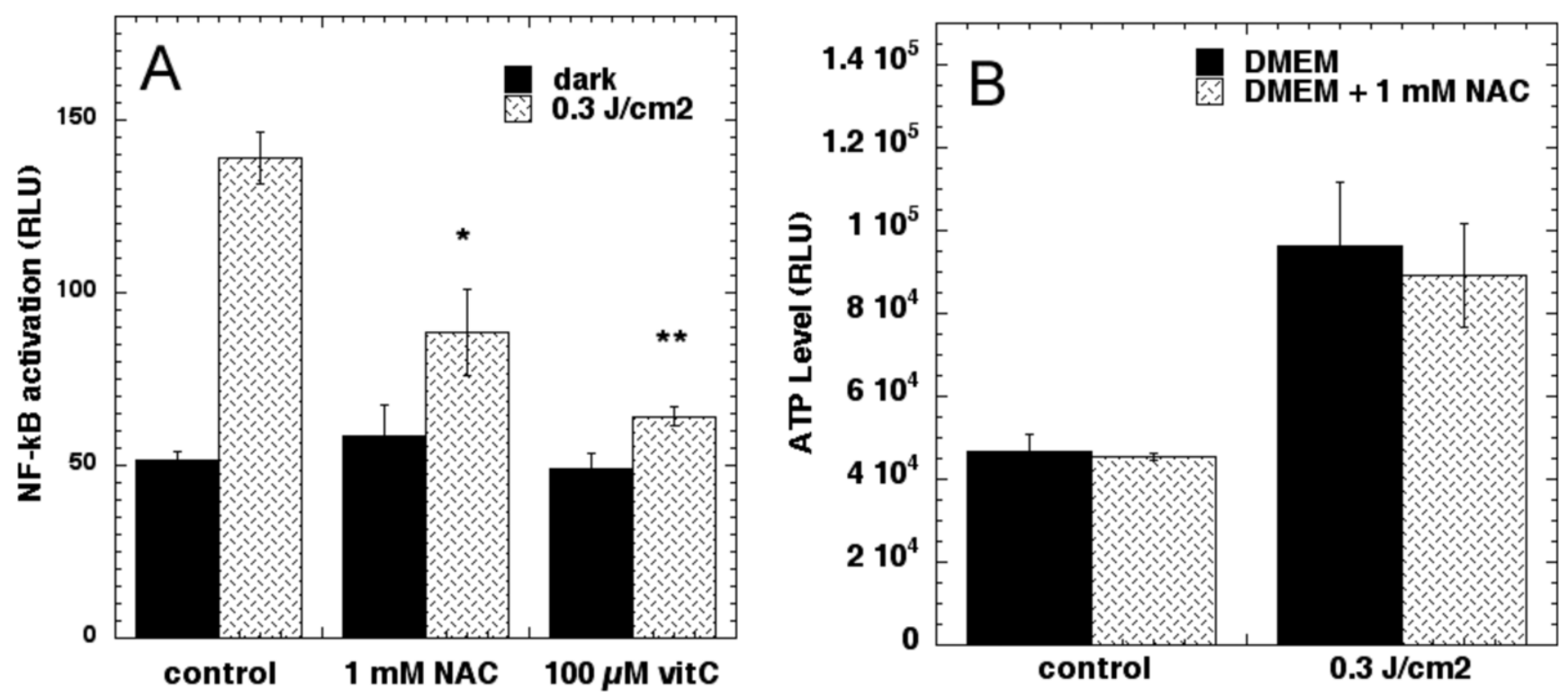

Figure 5. Antioxidants abrogate laser-induced NF-kB activation in MEF but not ATP increase. (A) NF-kB measured 6 hours post irradiation of cells incubated 2 hours with $1 \mathrm{mM} \mathrm{N}$-acetyl cysteine or $100 \mu \mathrm{M}$ ascorbic acid. (B) ATP measured after 2-hour incubation with 1 mM $\mathrm{N}$-acetyl cysteine. ${ }^{*} \mathrm{p}<0.05 ;{ }^{* *} \mathrm{p}<0.01$ compared to control.

doi:10.1371/journal.pone.0022453.g005 
is capable of oxidizing membrane lipids to lipid peroxides that then oxidize the dihydrofluorescein probe [41].

Karu et al. proposed a novel mitochondrial signaling pathway in mammalian cells initiated by red and near-IR light in vitro in 2004 [42]. One hypothesis to explain the effect of NIR light on cells is the absorption of the photons by cytochrome c oxidase $(\mathrm{CCO})$, which is unit IV of the respiratory chain $[6,42,43]$. The action spectrum of light on several cellular functions has been observed to closely resemble the absorption spectrum of $\mathrm{CCO}$ $[7,8]$. One theory that has yet to be conclusively be demonstrated is that nitric oxide can be photodissociated from CCO [44]. NO is known have a function as a regulator of respiration [45]. Hu et al. [46] showed that $\mathrm{He}-\mathrm{Ne}$ laser illumination increased mitochondrial membrane potential together with ATP synthesis in melanoma cells. They also observed upregulation of cytochrome c oxidase activity, increased phosphorylation of Jun $\mathrm{N}$ terminal kinase (JNK) that later activated activator protein-1 (AP1) which, in turn, led to increased cell proliferation. He-Ne laser irradiation in isolated mitochondria has been shown to promote $\mathrm{O}_{2}$ consumption by cytochrome c oxidase and increase electron transport [5].

NF-kB is a highly pleiotropic transcription factor [47] that induces expression of many gene products associated with known beneficial effects of LLLT. For instance anti-apoptotic and prosurvival proteins, proteins involved in cellular proliferation and migration and increased collagen synthesis and myofibroblast differentiation respond to NF-kB activation [48].

One interesting point that arises from our results is that NF-kB activation is known to be pro-inflammatory [49] while numerous reports indicate that LLLT has a pronounced anti-inflammatory effect. Recently Mafra de Lima et al reported [50] that the beneficial effects of LLLT in reducing inflammation in rat bronchial segments could be abrogated by a NF-kB inhibitor. A report by Moriyama et al [51] showed that LLLT increased inducible nitric oxide synthase expression in a mouse model of arthritis consistent with NF-kB activation. Another possibility is that the initial response to cell stress typical of NF-kB activation induces a productive response that includes lower NF-kB activation when measured at a later time point when the light has had a therapeutic effect. It is also possible that the initial proinflammatory response after LLLT induces expression of eicosanoids such as resolvins designed to end inflammation [52].

A limitation of our study is that we only showed laser-induced NF-kB activation in fibroblasts. It is possible that other cell types particularly those involved in inflammatory responses may show a different pattern of NF-kB activation after LLLT.

\section{Materials and Methods}

\section{Ethics statement}

All animal procedures were approved by the Subcommittee on Research Animal Care (IACUC) of Massachusetts General Hospital under protocol number 2009N000006 and met the guidelines of National Institutes of Health.

\section{Reagents}

2-Deoxy-L-glucose, sodium azide, cycloheximide, antimycin A, rotenone, $\mathrm{N}$-acetylcysteine, ascorbic acid, lipopolysaccharide from Escherichia coli and penicillin-streptomycin were from SigmaAldrich (St Louis, MO). Fluorescent probes 5-(and 6) chloromethyl-2',7'-dichlorodihydrofluorescein diacetate acetyl ester (CM- $\mathrm{H}_{2}$ DCFDA) and MitoTracker Red were from Invitrogen Molecular Probes (Invitrogen, Carlsbad, CA). Steady-Glo Luciferase Assay and Cell Titer Glo ATP Assay were from Promega
(Madison, WI). Bradford protein assay (BCA) was from Pierce Biotechnology Inc (Thermo-Scientific, Rockford, IL).

\section{Cell isolation and culture}

Timed pregnant female HLL (NF-kB luciferase reporter) mice [23] were used in the study. The animals were housed with a 12hour light/dark cycle with access to food and water ad libitum. Mouse embryonic fibroblasts (Luc MEF) were isolated from embryos removed from pregnant mice between day 13 and day 15 according to the protocol described by Sun and Taneja [53]. The cells were then cultured in Dulbecco's-modified Eagle's medium (DMEM) (Gibco, Invitrogen, Carlsbad, CA) supplemented with $10 \%$ fetal bovine serum (Hyclone, Waltham, MA) and $1 \%$ penicillin-streptomycin (Gibco, Invitrogen, Carlsbad, CA), in $37^{\circ} \mathrm{C}$ incubator. For all the experiments, cells were grown to about $80 \%$ confluence before seeding to 96 well plates and only the cells between passage 3 and passage 8 were employed.

\section{Laser irradiation}

The experiments were conducted with a diode laser (Model D030-MM-FCTS/B, Opto Power Corp, Tucson, AZ), which emits $810 \mathrm{~nm}$ near infrared radiation. Power densities generated in a round homogeneous spot of diameter 3-cm were measured with a power meter (Model DMM 199 with 201 Standard head, Coherent, Santa Clara, CA) and ranged from $1 \mathrm{~mW} / \mathrm{cm}^{2}$ to $30 \mathrm{~mW} / \mathrm{cm}^{2}$. Light was delivered for times varying from 7 seconds to 5 minutes to deliver different energy densities, namely 0.003 , $0.03,0.3,3$ and $30 \mathrm{~J} / \mathrm{cm}^{2}$. Wells were irradiated in blocks of $3 \mathrm{X}$ $3 \mathrm{cms}$ by use of a mask covering the plate.

\section{Assessing ROS generation}

Cells were plated in chamber slides (Nunc, Thermo-scientific, Rockford, IL) and incubated in $37^{\circ} \mathrm{C}$ incubator overnight, washed three times with PBS, and then changed to a PBS solution (with calcium and magnesium) (Cellgro, Manassas, VA) with $10 \mu \mathrm{M}$ CM- $\mathrm{H}_{2}$ DCFDA, $500 \mathrm{nM}$ MitoTracker Red and $300 \mathrm{nM}$ DAPI (Molecular Probes, Invtirogen, Carlsbad, CA) for $15 \mathrm{~min}$ and mounted on the microscope stage. In one experiment CM$\mathrm{H}_{2}$ DCFDA was added at different times after illumination and incubated for a further $30 \mathrm{~min}$ in every case to determine kinetics of ROS generation following laser irradiation (data not shown). Laser irradiation at various fluencies were performed on the samples and immediately imaged for fluorescence using an inverted fluorescence microscope (Olympus IX81, Centerway, PA) with a spinning disc laser system (BD Carv II, BD Biosciences, Franklin Lakes, NJ) with equal exposure times and images were collected with IP lab (Ver 4.0, Exton, PA).

For Quantitative ROS measurements, the Amplex UltraRed (Moelcular Probes, Invitrogen, Carlsbad, CA) was used. Briefly, the cells were seeded in a 96 well black walled plate with clear bottoms (Costar, Corning, NY) and laser irradiated at varying doses followed by addition of PBS (with calcium and magnesium) (Cellgro, Manassas, VA) with $100 \mu \mathrm{M}$ Amplex Ultrared and $0.2 \mathrm{U} / \mathrm{ml}$ Horse radish peroxidase (both Molecular Probes, Invtirogen, Carlsbad, CA) for $30 \mathrm{~min}$. Fluorescence was measured using a microplate reader (Synergy HT, Bio-Tek, Winooski, VT).

\section{Luciferase Assay for NF-kB activation}

MEFs were plated in a 96 well plates (Costar, Corning, NY) with 3000 to 5000 cells per well and were treated with either LLLT or $0.5 \mu \mathrm{g} / \mathrm{mL}$ LPS, washed with PBS and at lysed with $50 \mu \mathrm{l}$ passive lysis buffer (Promega, Madison, WI) at various time points post treatment. Lysates were transferred to 96 well white 
plates and substrate was added (Promega, Madison, WI) to assess luciferase activity with a microplate reader (Synergy HT, Bio-Tek, Winooski, VT). The remaining lysate was used in a Bradford assay (BCA Assay, Pierce Biotechnology Inc, Thermo-Scientific, Rockford, IL) to estimate total protein content for normalization. Cycloheximide $(10 \mu \mathrm{M})$ (Calbiochem, EMD Biochemicals, Gibbstown, $\mathrm{NJ}$ ) was added 2 hours before treatments to inhibit protein synthesis.

\section{Immunoblotting}

Following laser irradiation, cells were washed with cold PBS and lysed $(175 \mathrm{mM} \mathrm{NaCl}, 25 \mathrm{mM}$ HEPES pH 7.4, 10\% Glycerol, $5 \mathrm{mM}$ EDTA, 1\% Triton X, $50 \mathrm{mM}$ Sodium Fluoride, $5 \mathrm{mM}$ Sodium Orthovandate) with Complete Mini Protease inhibitor (Roche). The cells were then scarped using a plastic cell lifter, transferred to labeled microfuge tubes and spun at $14000 \mathrm{rpm}$ at $4^{\circ} \mathrm{C}$ for $15 \mathrm{~min}$. The clear supernatant was transferred to a fresh tube and total protein was estimated by Bradford assay (BCA Assay, Pierce Biotechnology Inc, Thermo-Scientific, Rockford, IL) and electrophoresis was performed with precast Tris-Glycine gels (Invitrogen, Carlsbad, CA), transferred onto $0.2 \mu \mathrm{m}$ nitrocellulose membranes (Invitrogen, Carlsbad, CA), blocked with 3\% bovine serum albumin (Sigma-Aldrich, St Louis, MO) and incubated with primary antibodies Phospho-NFkB (Ser 536), Phospho IkB $\alpha / \beta$ (both Cell Signaling, Beverly, MA) and Actin (Chemicon) overnight at $4 \mathrm{C}$ followed by appropriate species-specific secondary antibody (Jackson ImmunoResearch Laboratories Inc, West Groove, PA). Following rigorous washes with Tris Buffered Saline and Tween, chemiluminescence substrate (Pierce Biotechnology Inc, Thermo-Scientific, Rockford, IL) was added to the membrane and detected by films (Kodak MR, Sigma-Aldrich, St Louis, MO).

\section{References}

1. Tunér J, Hode L (2002) Laser therapy, clinical practice and scientific background. Grängesberg, ed. Sweden: Prima Books.

2. Karu TI (1998) The science of low power laser therapy. London, UK: Gordon and Breach Scientific Publications.

3. Karu T (1989) Photobiology of low-power laser effects. Health Phys 56: 691-704.

4. Szundi I, Liao GL, Einarsdottir O (2001) Near-infrared time-resolved optical absorption studies of the reaction of fully reduced cytochrome c oxidase with dioxygen. Biochemistry 40: 2332-2339.

5. Pastore D, Greco M, Passarella S (2000) Specific helium-neon laser sensitivity of the purified cytochrome c oxidase. Int J Radiat Biol 76: 863-870.

6. Karu T (1999) Primary and secondary mechanisms of action of visible to nearIR radiation on cells. J Photochem Photobiol B 49: 1-17.

7. Karu TI, Kolyakov SF (2005) Exact action spectra for cellular responses relevant to phototherapy. Photomed Laser Surg 23: 355-361.

8. Wong-Riley MT, Liang HL, Eells JT, Chance B, Henry MM, et al. (2005) Photobiomodulation directly benefits primary neurons functionally inactivated by toxins: role of cytochrome c oxidase. J Biol Chem 280: 4761-4771.

9. Zhang Y, Song S, Fong CC, Tsang CH, Yang Z, et al. (2003) cDNA microarray analysis of gene expression profiles in human fibroblast cells irradiated with red light. J Invest Dermatol 120: 849-857.

10. Wang T, Zhang X, LiJJ (2002) The role of NF-kappaB in the regulation of cell stress responses. Int Immunopharmacol 2: 1509-1520.

11. Baichwal VR, Baeuerle PA (1997) Activate NF-kappa B or die? Curr Biol 7: R94-96.

12. Henkel T, Machleidt T, Alkalay I, Kronke M, Ben-Neriah Y, et al. (1993) Rapid proteolysis of I kappa B-alpha is necessary for activation of transcription factor NF-kappa B. Nature 365: 182-185.

13. Hoffmann A, Levchenko A, Scott ML, Baltimore D (2002) The IkappaB-NFkappaB signaling module: temporal control and selective gene activation. Science 298: 1241-1245.

14. D'Angio CT, Finkelstein JN (2000) Oxygen regulation of gene expression: a study in opposites. Mol Genet Metab 71: 371-380.

15. Li N, Karin M (1999) Is NF-kappaB the sensor of oxidative stress? Faseb J 13: 1137-1143.

16. Schreck R, Rieber P, Baeuerle PA (1991) Reactive oxygen intermediates as apparently widely used messengers in the activation of the NF-kappa B transcription factor and HIV-1. Embo J 10: 2247-2258.

\section{Cell-Titer Glo Assay for ATP}

MEFs were plated in a 96 well plates (Costar, Corning, NY) with 3000 to 5000 cells per well. At the various time points following LLLT, $50 \mu \mathrm{L}$ cell lysis buffer was added and plates were placed on shaker for 2 minutes to ensure completely release of ATP. $95 \mu$ lysates were then transferred to luminescence 96 well plates (White plate, Costar, Corning, NY) and $100 \mu \mathrm{L}$ of CellTiter Glo Assay mix was added into each well, shaken for 5 minutes to stabilize the luminescence signal and measured with a plate luminometer (Wallac Tri-Lux beta, PerkinElmer Life and Analytical Sciences, Waltham, MA). $5 \mu \mathrm{l}$ of the lysate was used to estimate total protein (BCA Assay, Pierce Biotechnology Inc, Thermo-Scientific, Rockford, IL). Another set of cells was incubated with $5 \mathrm{mM}$ 2-deoxy-L-glucose and $0.05 \%$ sodium azide for two hours before laser irradiation to inhibit ATP synthesis. In some experiments, antioxidants $\mathrm{N}$-acetylcysteine $(1 \mathrm{mM})$ and ascorbic acid $(100 \mu \mathrm{M})$ were added for 2 hours before laser irradiation.

\section{Statistical Analysis}

All assays were performed in multiple wells $(n=9)$ and Microsoft Excel (Redmond, WA) software was used for SingleFactor ANOVA to evaluate the statistical significance of experimental results $(\mathrm{p}<0.05)$.

\section{Author Contributions}

Conceived and designed the experiments: PRA DM MRH. Performed the experiments: AC-HC PRA YYH EMT SKS GBK TS. Analyzed the data: PRA MRH. Contributed reagents/materials/analysis tools: FEY TSB. Wrote the paper: AC-HC PRA MRH.
17. Schreck R, Grassmann R, Fleckenstein B, Baeuerle PA (1992) Antioxidants selectively suppress activation of NF-kappa B by human T-cell leukemia virus type I Tax protein. J Virol 66: 6288-6293.

18. Eichler M, Lavi R, Friedmann H, Shainberg A, Lubart R (2007) Red lightinduced redox reactions in cells observed with TEMPO. Photomed Laser Surg 25: $170-174$.

19. Lubart R, Eichler M, Lavi R, Friedman H, Shainberg A (2005) Low-energy laser irradiation promotes cellular redox activity. Photomed Laser Surg 23: 3-9.

20. Alexandratou E, Yova D, Handris P, Kletsas D, Loukas S (2002) Human fibroblast alterations induced by low power laser irradiation at the single cell level using confocal microscopy. Photochem Photobiol Sci 1: 547-552.

21. Pal G, Dutta A, Mitra K, Grace MS, Romanczyk TB, et al. (2007) Effect of low intensity laser interaction with human skin fibroblast cells using fiber-optic nanoprobes. J Photochem Photobiol B 86: 252-261.

22. Tafur J, Mills PJ (2008) Low-intensity light therapy: Exploring the role of redox mechanisms. Photomed Laser Surg epub ahead of print.

23. Blackwell TS, Yull FE, Chen CL, Venkatakrishnan A, Blackwell TR, et al. (2000) Multiorgan nuclear factor kappa B activation in a transgenic mouse model of systemic inflammation. Am J Respir Crit Care Med 162: 1095-1101.

24. Sadikot RT, Jansen ED, Blackwell TR, Zoia O, Yull F, et al. (2001) High-dose dexamethasone accentuates nuclear factor-kappa $\mathrm{b}$ activation in endotoxintreated mice. Am J Respir Crit Care Med 164: 873-878.

25. Callaghan GA, Riordan C, Gilmore WS, McIntyre IA, Allen JM, et al. (1996) Reactive oxygen species inducible by low-intensity laser irradiation alter DNA synthesis in the haemopoietic cell line U937. Lasers Surg Med 19: 201-206.

26. Grossman N, Schneid N, Reuveni H, Halevy S, Lubart R (1998) 780 nm low power diode laser irradiation stimulates proliferation of keratinocyte cultures: involvement of reactive oxygen species. Lasers Surg Med 22: 212-218.

27. Lavi R, Shainberg A, Friedmann H, Shneyvays V, Rickover O, et al. (2003) Low energy visible light induces reactive oxygen species generation and stimulates an increase of intracellular calcium concentration in cardiac cells. J Biol Chem 278: 40917-40922.

28. Yu W, Naim JO, McGowan M, Ippolito K, Lanzafame RJ (1997) Photomodulation of oxidative metabolism and electron chain enzymes in rat liver mitochondria. Photochem Photobiol 66: 866-871.

29. Zhang J, Xing D, Gao X (2008) Low-power laser irradiation activates Src tyrosine kinase through reactive oxygen species-mediated signaling pathway. J Cell Physiol 217: 518-528. 
30. Schroeder P, Pohl C, Calles C, Marks C, Wild S, et al. (2007) Cellular response to infrared radiation involves retrograde mitochondrial signaling. Free Radic Biol Med 43: 128-135.

31. Glascott PA, Jr., McSorley KM, Mittal B, Sanger JM, Sanger JW (1987) Stress fiber reformation after ATP depletion. Cell Motil Cytoskeleton 8: 118-129.

32. Deng YT, Huang HC, Lin JK (2009) Rotenone induces apoptosis in MCF-7 human breast cancer cell-mediated ROS through JNK and p38 signaling. Mol Carcinog.

33. Park WH, Han YW, Kim SH, Kim SZ (2007) An ROS generator, antimycin A, inhibits the growth of HeLa cells via apoptosis. J Cell Biochem 102: 98-109.

34. Ali S, Jain SK, Abdulla M, Athar M (1996) Paraquat induced DNA damage by reactive oxygen species. Biochem Mol Biol Int 39: 63-67.

35. Arany PR, Nayak RS, Hallikerimath S, Limaye AM, Kale AD, et al. (2007) Activation of latent TGF-betal by low-power laser in vitro correlates with increased TGF-betal levels in laser-enhanced oral wound healing. Wound Repair Regen 15: 866-874.

36. Hauer J, Puschner S, Ramakrishnan P, Simon U, Bongers M, et al. (2005) TNF receptor (TNFR)-associated factor (TRAF) 3 serves as an inhibitor of TRAF2/5mediated activation of the noncanonical NF-kappaB pathway by TRAF-binding TNFRs. Proc Natl Acad Sci U S A 102: 2874-2879.

37. Senftleben U, Cao Y, Xiao G, Greten FR, Krahn G, et al. (2001) Activation by IKKalpha of a second, evolutionary conserved, NF-kappa B signaling pathway. Science 293: 1495-1499.

38. Storz P (2007) Mitochondrial ROS-radical detoxification, mediated by protein kinase D. Trends Cell Biol 17: 13-18.

39. Storz P, Toker A (2003) Protein kinase D mediates a stress-induced NF-kappaB activation and survival pathway. Embo J 22: 109-120.

40. Storz P, Doppler H, Toker A (2005) Protein kinase D mediates mitochondrionto-nucleus signaling and detoxification from mitochondrial reactive oxygen species. Mol Cell Biol 25: 8520-8530.

41. Bilski P, Belanger AG, Chignell CF (2002) Photosensitized oxidation of 2', 7'dichlorofluorescin: singlet oxygen does not contribute to the formation of fluorescent oxidation product $2^{\prime}, 7^{\prime}$-dichlorofluorescein. Free Radic Biol Med 33: 938-946.
42. Karu TI, Pyatibrat LV, Afanasyeva NI (2004) A novel mitochondrial signaling pathway activated by visible-to-near infrared radiation. Photochem Photobiol 80: $366-372$.

43. Karu TI, Pyatibrat LV, Kolyakov SF, Afanasyeva NI (2005) Absorption measurements of a cell monolayer relevant to phototherapy: Reduction of cytochrome c oxidase under near IR radiation. J Photochem Photobiol B 81: 98-106.

44. Lane N (2006) Cell biology: power games. Nature 443: 901-903.

45. Shiva S, Brookes PS, Patel RP, Anderson PG, Darley-Usmar VM (2001) Nitric oxide partitioning into mitochondrial membranes and the control of respiration at cytochrome c oxidase. Proc Natl Acad Sci U S A 98: 7212-7217.

46. Hu WP, Wang JJ, Yu CL, Lan GC, Chen GS, et al. (2007) Helium-neon laser irradiation stimulates cell proliferation through photostimulatory effects in mitochondria. J Invest Dermatol 127: 2048-2057.

47. Tergaonkar V (2006) NFkappaB pathway: a good signaling paradigm and therapeutic target. Int J Biochem Cell Biol 38: 1647-1653.

48. Campbell KJ, Perkins ND (2006) Regulation of NF-kappaB function. Biochem Soc Symp. pp 165-180.

49. Carmody RJ, Chen YH (2007) Nuclear factor-kappaB: activation and regulation during toll-like receptor signaling. Cell Mol Immunol 4: 31-41.

50. Mafra de Lima F, Naves KT, Machado AH, Albertini R, Villaverde AB, et al (2009) Lung inflammation and endothelial cell damage are decreased after treatment with phototherapy $(\mathrm{PhT})$ in a model of acute lung injury induced by Escherichia coli lipopolysaccharide in the rat. Cell Biol Int 33: 1212-1221.

51. Moriyama Y, Moriyama EH, Blackmore K, Akens MK, Lilge L (2005) In vivo study of the inflammatory modulating effects of low-level laser therapy on iNOS expression using bioluminescence imaging. Photochem Photobiol 81: 1351-1355.

52. Serhan CN, Chiang N, Van Dyke TE (2008) Resolving inflammation: dual antiinflammatory and pro-resolution lipid mediators. Nature reviews Immunology 8: 349-361.

53. Sun H, Taneja R (2007) Analysis of transformation and tumorigenicity using mouse embryonic fibroblast cells. Methods Mol Biol 383: 303-310. 